\title{
Ultrasound-Guided Miniscalpel-Needle Release versus Dry Needling for Chronic Neck Pain: A Randomized Controlled Trial
}

\author{
Yongjun Zheng, ${ }^{1}$ Dongping Shi, ${ }^{2}$ Xiaotong Wu, ${ }^{3}$ Minghong Gu, ${ }^{4}$ Zisheng Ai, \\ Kun Tang, ${ }^{6}$ Le Ye, ${ }^{1}$ and Xiangrui Wang ${ }^{1}$ \\ ${ }^{1}$ Department of Pain Management, Renji Hospital, School of Medicine, Shanghai Jiao Tong University, 1630 Dongfang Road, \\ Shanghai 200127, China \\ ${ }^{2}$ Department of Anesthesiology, Shanghai Jiading Central Hospital, Shanghai 201800, China \\ ${ }^{3}$ Department of Anesthesiology, Shanghai First Rehabilitation Hospital, Shanghai 200090, China \\ ${ }^{4}$ Department of Pain Management, Shanghai Jiading Hospital of Traditional Chinese Medicine, Shanghai 201800, China \\ ${ }^{5}$ Department of Preventive Medicine, College of Medicine, Tongji University, Shanghai 200092, China \\ ${ }^{6}$ Department of Anesthesiology, Tongren Hospital, School of Medicine, Shanghai Jiao Tong University, Shanghai 200336, China
}

Correspondence should be addressed to Xiangrui Wang; wangxiangrui2012@163.com

Received 22 April 2014; Revised 5 September 2014; Accepted 9 September 2014; Published 16 October 2014

Academic Editor: Lixing Lao

Copyright (C) 2014 Yongjun Zheng et al. This is an open access article distributed under the Creative Commons Attribution License, which permits unrestricted use, distribution, and reproduction in any medium, provided the original work is properly cited.

Objective. To compare ultrasound-guided miniscalpel-needle (UG-MSN) release versus ultrasound-guided dry needling (UG-DN) for chronic neck pain. Methods. A total of 169 patients with chronic neck pain were randomized to receive either UG-MSN release or UG-DN. Before treatment and at 3 and 6 months posttreatment, pain was measured using a 10-point visual analogue scale (VAS). Neck function was examined using the neck disability index. Health-related quality of life was examined using the physical component score (PCS) and mental component score (MCS) of the SF-36 health status scale. Results. Patients in the UG-MSN release had greater improvement on the VAS (by 2 points at 3 months and 0.9 points at 6 months) versus in the UG-DN arm; (both $P<0.0001)$. Patients receiving UG-MSN release also showed significantly lower scores on the adjusted neck disability index, as well as significantly lower PCS. No severe complications were observed. Conclusion. UG-MSN release was superior to UG-DN in reducing pain intensity and neck disability in patients with chronic neck pain and was not associated with severe complications. The procedural aspects in the two arms were identical; however, we did not verify the blinding success. As such, the results need to be interpreted with caution.

\section{Introduction}

Chronic neck pain is a common and disabling condition $[1,2]$. It usually originates in discrete, hyperirritable focal spots (trigger points) in a taut band of skeletal muscle; onset of pain often occurs in conjunction with musculoskeletal disorders [3]. Injecting analgesic medication directly into trigger points could provide short-term relief of the symptoms [3]. However, several studies suggested that dry needling (DN; simply inserting a needle into trigger points) can also provide pain relief [4-6]. In fact, $\mathrm{DN}$ of primary trigger points has been shown to inhibit activity at satellite trigger points [7]. A meta-analysis, however, has concluded that $\mathrm{DN}$ does not provide significant therapeutic effect relative to placebo [8].

Miniscalpel-needle (MSN) release is a promising treatment for chronic neck pain [9-11]. In this technique, a small needle-scalpel is used to stimulate areas of chronic injury or soft tissue damage [9]. In one study [9], MSN release led to significantly greater reduction in pain intensity than did acupuncture, as well as significantly greater increases in pain threshold and motion range at 3 months after treatment [9].

A major concern of the conventional MSN release is the potential damage to nerves and blood vessels [12,13]. Limited evidence suggests that performing the MSN procedure under 
the guidance of ultrasound could help to avoid damaging critical nerves and vessels in unexpected locations [14-16].

The current study is a randomized controlled trial (RCT) that compared MSN release and dry needling in patients with chronic neck pain.

\section{Subjects and Methods}

2.1. Subjects. This study was approved by the Institutional Review Board of Renji Hospital (Shanghai, China) and registered in the Chinese Clinical Trial Register (ChiCTR-TRC-10001609). Data collection, analysis, and reporting were performed in compliance with the CONSORT guidelines [17]. Written informed consent was obtained from all participants.

Consecutive adult ( $\geq 18$ years old) outpatients with chronic neck pain seeking medical treatment at the Department of Pain Management, Renji Hospital, from January 2009 to February 2010 were recruited. Eligibility was determined at the preliminary interview. For inclusion in the study, patients had to meet all the following criteria: chronic neck pain for more than three months; a score $>3$ on a 10point visual analogue scale (VAS); and the presence of trigger points in the neck. Trigger points were established based on both physical signs (hypersensitive bundle or nodule of muscle fibers harder than normal upon palpation) and ultrasound imaging (hyperechoic skin, hyperechoic marbled appearance of the muscle, and mixed echogenicity). Patients were excluded if they were pregnant or if they had a history of acupuncture, DN, MSN release, or vertebral column surgery. They were also excluded if they had any of the following conditions: protrusion or prolapse of one or more intervertebral discs with concurrent neurological symptoms; infectious spondylopathy; chronic neck pain caused by inflammatory, malignant, or autoimmune disease; congenital deformation of spine except slight lordosis or scoliosis; compression fracture caused by osteoporosis; spinal stenosis and spondylolysis or spondylolisthesis.

2.2. Pain Management. Patients were randomly assigned to receive either UG-MSN release or UG-DN using a block design $(n=8)$. The allocation concealment was carried out using the SNOSE method (sequentially numbered, opaque, sealed envelopes). UG-MSN was performed using an MSN (Shenlong Medical Apparatus, Suzhou, China; see Supplementary Figures 1(a) and 1(b) in the Supplementary Material available online at http://dx.doi.org/10.1155/2014/235817) as described earlier $[9,10]$ (Supplementary Figure 2(a)). The ultrasound system (SonoSite, Bothell, Washington, USA) comprised a high-frequency linear array transducer (10$5 \mathrm{MHz}$ ) covered by a sterilized medical condom as a disposable sterile medical ultrasound sheath (Shanghai Medical Dressing Factory, Shanghai, China; Supplementary Figures 1(c) and $1(\mathrm{~d})$ ).

With the patient in a lateral decubitus position, trigger points were identified by palpation by one author (YJZ) and marked with indelible ink. The skin was sterilized by povidone iodine. The transducer was applied transversely to the lateral aspect of the neck to obtain a transverse axial view. The seventh cervical vertebra (C7) was used as a reference and identified by the rudimentary anterior tubercle and a prominent posterior tubercle on the transverse process (Figures 2(a)-2(c)) [16]. By moving the transducer cranially, C6 was easily identified (Figure 3 ). Trigger points were identified by the following features: hyperechoic skin, adipose tissue of mixed echogenicity, and hyperechoic marbled appearance of muscle (Figure 3(c)) [18]. After confirming the trigger points, intradermal anesthesia with $1 \%$ lidocaine was performed using a 25-gauge, 1.5 -inch needle and $2 \mathrm{~mL}$ syringe, and either UG-MSN release or UG-DN was performed on each point.

One author (YJZ) performed the treatment (either MSN release or DN under ultrasound guidance). For MSN release, when the pinpoint needle reached the trigger point, the MSN was moved up and down 2-3 times longitudinally around the vertebral lamina and the posterior tubercle of the transverse processes of C6 (Figure 3(c)). The miniscalpel did not extend deeper than the posterior tubercle of the transverse processes of the cervical vertebrae, thereby avoiding important structures between the posterior and anterior tubercles of the cervical vertebrae [19]. MSN release was performed once for each trigger point. For UG-DN, the needle was inserted in the trigger point, longitudinal to the transducer at the level of C6 ((Supplementary Figure 2(b)) and Figure 4). Needle placement was verified by asking the patient to contract the muscle and observing the needle under ultrasound. The needle was moved up and down 2-3 times. Again, the needle was inserted no deeper than the posterior tubercle of the transverse processes of the cervical vertebrae. DN was performed once for each trigger point.

2.3. Outcomes. The primary outcome was pain intensity. Secondary outcomes included pain disability and healthrelated quality of life. Pain intensity was measured using the 10-point VAS [18]. Pain disability was measured using the neck disability index (NDI), consisting of 10 questions with six possible responses, each of which was assigned a different number of points. The total NDI score can range from 0 to 50 points, with 50 indicating the worst condition $[20,21]$. Health-related quality of life was measured using the SF-36 health status scale $[22,23]$.

The survey instruments were administered by a pain management specialist blinded to the treatment (even whether a patient was in the study or not). Outcomes were assessed before the treatment and at 3 and 6 months after the treatment.

2.4. Sample Size. Sample size was calculated based on a preliminary RCT involving 60 patients with chronic neck pain, 30 of whom were treated by UG-MSN release and 30 by UG-DN. None of the 60 subjects in this preliminary study was included in the current study. Sample size $(n)$ was calculated according to the following equation for repeated measures at a significance level of 0.05 and power of $80 \%: n=$ $2 \times\left(\mu_{\alpha} / 2+\mu_{\beta}\right)^{2} \times \delta^{2} \times(1-\rho) /\left(m \times s^{2} \times\left(\beta_{1 A}-\beta_{1 B}\right)^{2}\right)\left(\mu_{\alpha} / 2=\right.$ $\left.1.96, \mu_{\beta}=0.842\right)$ [24]. Based on the results of the preliminary 
study, the variance of repeated measurements $\left(\delta^{2}\right)$ was 3.08; the symmetric correlation coefficient $(\rho)$ was 0.38 ; the number of repetitions $(m)$ was $3 ; s^{2}=\sum\left(t_{j}-t\right)^{2} / m=8.25$, where $t_{j}$ is the time when repeated measures are performed and $t$ is the average time of repeated measures; and $\beta_{1 A}-\beta_{1 B}=0.137$, where $\beta_{1 A}$ and $\beta_{1 B}$ are the slopes (change in measurement over time) for the UG-MSN and UG-DN groups, respectively. This equation indicated the need for 64 participants per arm, so we recruited 80 patients for each arm to take into account a $20 \%$ dropout rate.

2.5. Randomization. Subjects were randomly allocated into UG-MSN or UG-DN group based on a computer-generated allocation sequence using a block design $(n=8)$. Allocation concealment was carried out using the SNOSE method (sequentially numbered, opaque, sealed envelopes). The next envelope in the sequence was opened after the participant had given informed consent.

2.6. Blinding. Patients were blinded to their treatment allocation and did not see the instruments during the procedure. The pain management specialist who conducted assessment was also blinded to the treatment condition. The same physician (YJZ) performed UG-MSN and UG-DN procedures on all participants. Data were analyzed by an independent statistician.

2.7. Statistical Analysis. All data were analyzed using SPSS 13.0 (IBM, Chicago, USA). Data for continuous variables showing a normal distribution were reported as means and standard deviations (SD); otherwise, data were reported as median. Intergroup differences were assessed for statistical significance using independent Student's $t$-test for normally distributed data and the Mann-Whitney test for nonnormally distributed data. Intergroup differences in categorical variables were assessed for significance using the $\chi^{2}$ test or Fisher's exact test. Analysis of covariance was also used to assess differences between the two groups based on $F$ statistics. The baseline served as a covariate. The significance level for all tests was set at 0.05 .

\section{Results}

3.1. Participant Flow. From January 2009 to February 2010, 250 participants were approached, of which 81 were excluded, leaving 169 who were randomized into UG-MSN or UGDN group (Figure 1). Each group received the corresponding treatment once a week for three weeks. Of the 169 patients, $14(8.3 \%)$ dropped out, and the dropout rate was similar for the UG-MSN release and UG-DN groups (6.8\% versus $9.9 \%$, $P=0.5801, \chi^{2}$ test). In the UG-MSN group, 4 patients were lost to follow-up by 3 months posttreatment and 6 were lost by 6 months. In the UG-DN group, 4 patients were lost by 3 months and 8 were lost by 6 months.

Scores on the VAS, NDI, SF-36 PCS, and SF-36 MCS were measured immediately prior to the start of treatment and again at 3 and 6 months posttreatment. VAS scores at baseline were similar for the patients lost to follow-up from either group at 3 months and 6 months $(P>0.05$, Mann-Whitney nonparametric tests). This suggests that losses to follow-up did not significantly affect the outcomes measured for the patients who completed the trial.

3.2. Baseline Characteristics. Baseline demographic data, disease duration, previous treatments, and scores on the VAS, NDI, SF-36 PCS, and SF-36 MCS are shown in Table 1. None of these parameters differed significantly between the two treatment groups.

3.3. Outcome Measures. The final analysis included data for 82 of 88 patients $(93.2 \%)$ in the UG-MSN release group and for 73 of 81 patients $(90.1 \%)$ in the UG-DN group. Analyses of covariance were performed in which the score on the VAS, NDI, SF-36 PCS, or SF-36 MCS at 3 and 6 months served as the response variable (outcome) and treatment served as the explanatory variable; results were adjusted for baseline differences in the response variable.

At 3 months posttreatment, the adjusted mean VAS was 0.9 points higher in the UG-DN group than in the UG-MSN release group $(\beta=0.9, \mathrm{SD}=0.2, P<0.0001)$; at 6 months, this difference had grown to 2.0 points $(\beta=2.0, \mathrm{SD}=0.2$, $P<0.0001$; Table 2). Group-level analysis showed that mean VAS decreased by $51.5 \%$ from baseline to 3 months in the UGMSN release group, compared to $40.8 \%$ in the UG-DN group. Mean VAS decreased by $44.1 \%$ from baseline to 6 months in the UG-MSN release group, compared to $18.3 \%$ in the UGDN group. Patient-level analysis showed that 50 of 84 patients in the UG-MSN release group showed a $>50 \%$ reduction in VAS from baseline to 3 months, compared to 38 of 84 patients in the UG-DN group. The corresponding proportions at 6 months were 35 of 82 patients in the UG-MSN release group, compared to 15 of 82 patients in the UG-DN group.

At 3 months posttreatment, the adjusted average NDI was 2.4 points higher in the UG-DN group than in the UG-MSN release group $(\beta=2.4, \mathrm{SD}=0.7, P<0.0001)$; at 6 months, this difference had grown to 5.3 points $(\beta=5.3, \mathrm{SD}=0.7, P<$ 0.0001 ; Table 2). Group-level analysis showed that mean NDI decreased by $64.3 \%$ from baseline to 3 months in the UGMSN release group, compared to $45 \%$ in the UG-DN group. Mean NDI decreased by $60.3 \%$ from baseline to 6 months in the UG-MSN release group, compared to $23.8 \%$ in the UGDN group. Patient-level analysis showed that 55 of 84 patients in the UG-MSN release group showed a $>50 \%$ reduction from baseline to 3 months, compared to 40 of 84 patients in the UG-DN group. The corresponding proportions at 6 months were 50 of 82 patients in the UG-MSN release group, compared to 17 of 82 patients in the UG-DN group.

At 3 months posttreatment, the adjusted mean SF-36 PCS was 5.5 points lower in the UG-DN group than in the UGMSN release group $(\beta=5.5, \mathrm{SD}=2.2, P=0.013)$; at 6 months, this difference had grown to 7.5 points $(\beta=7.5$, $\mathrm{SD}=3.3, P=0.024$; Table 2 ). Group-level analysis showed that mean SF-36 PCS improved by $19.1 \%$ from baseline to 3 months in the UG-MSN release group, compared to $1.1 \%$ in the UG-DN group. Mean SF-36 PCS improved by $33.4 \%$ from baseline to 6 months in the UG-MSN release group, 


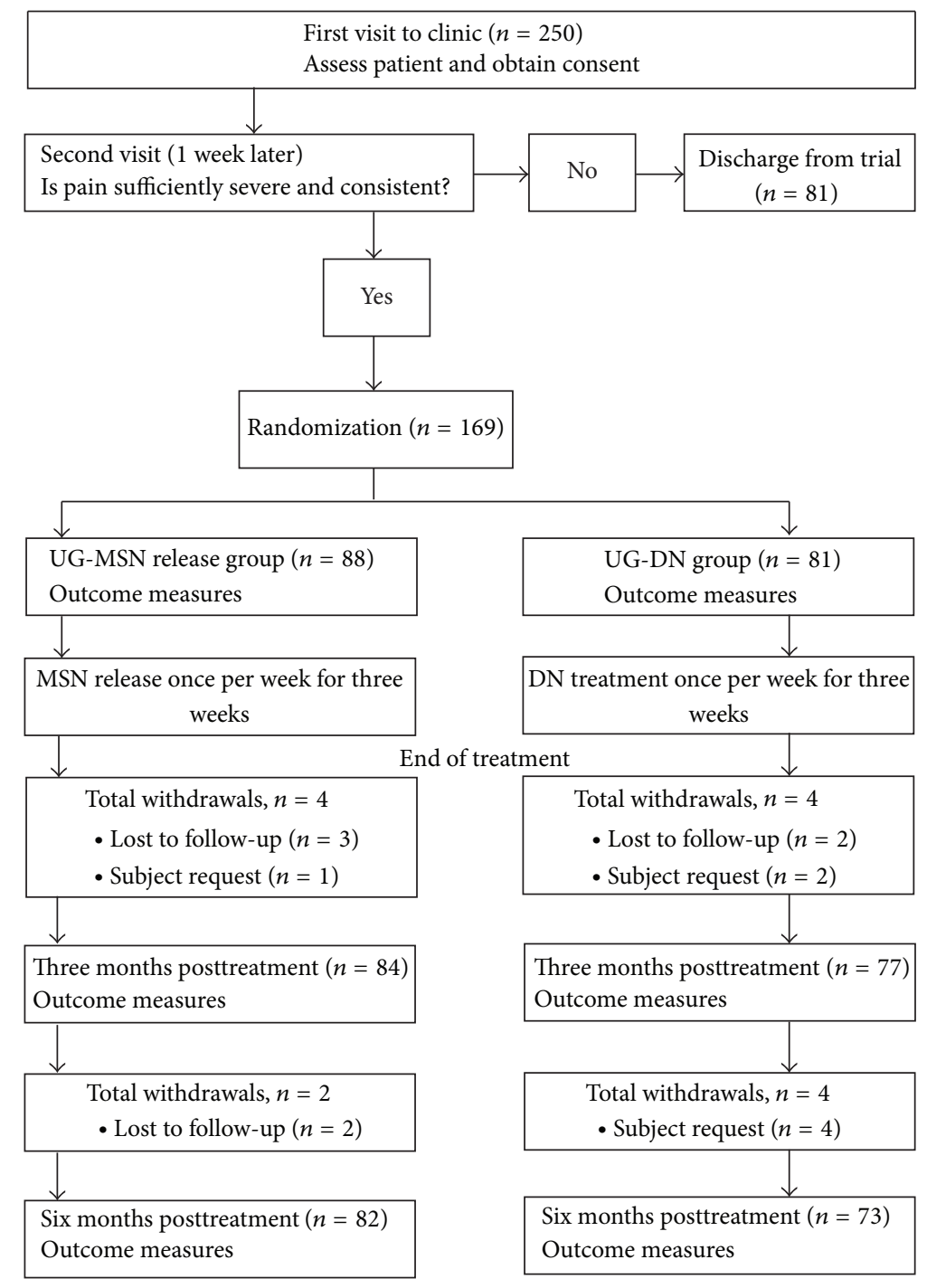

FIGURE 1: Schematic presentation of the study flow.

compared to $9.9 \%$ in the UG-DN group. However, the two treatment groups showed similar SF-36 MCS scores at 6 months.

3.4. Treatment Adherence. Treatment adherence rates were similar between the two treatment groups: 82 of 88 subjects (93.2\%) in the UG-MSN release group completed the study protocol, compared to 73 of $81(90.1 \%)$ in the UG-DN group $\left(P=0.5801, \chi^{2}\right.$ test $)$.

3.5. Adverse Events. Six participants in the UG-MSN release group (7.3\%) and 7 in the UG-DN group (9.6\%) reported mild reactions during treatment, mainly slight pain and somatic reactions such as sweating. However, all these participants agreed to continue the treatment. No serious adverse complications, such as spinal injury or nerve injury, were recorded.

\section{Discussion}

The results of this randomized trial suggest that UG-MSN release is more effective than UG-DN in reducing pain intensity and neck disability and in increasing health-related quality of life in patients with chronic neck pain. Both treatments appear to be associated with similarly low rates of mild adverse events, similar dropout rates, and no severe side effects. These findings should be confirmed in larger cohorts. To our knowledge, this is the first report using ultrasound to guide MSN release.

The greater efficacy of UG-MSN may reflect the fact that it combines the therapeutic action of DN and minimally invasive surgery. MSN release can cut and detach the taut band in myofascial pain syndrome as well as relaxing the compressed blood vessels and trapped nerves at the primary and second foci [25]. MSN release also stimulates vessel and nerve bundles, restores mechanical dynamic equilibrium, 


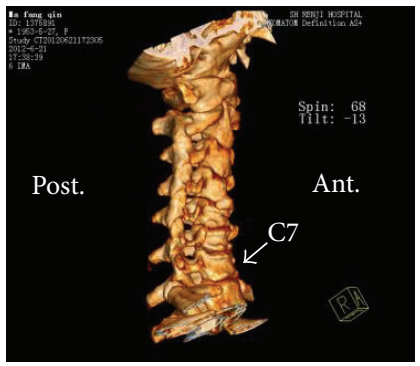

(a)

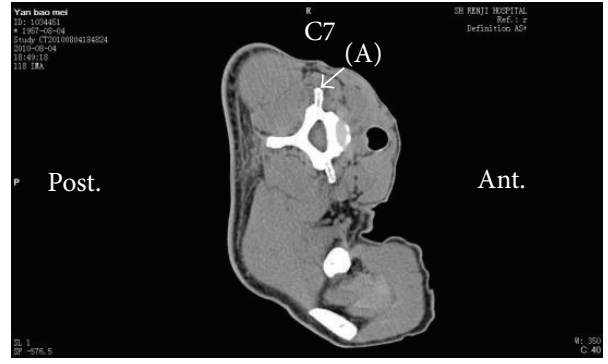

(b)

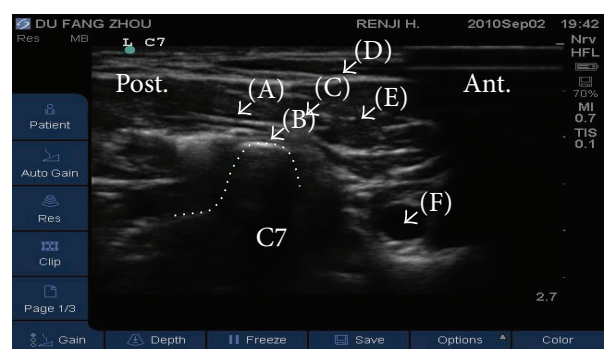

(c)

FIGURE 2: Ultrasound identification of the seventh cervical vertebra (C7) during the UG-MSN and UG-DN techniques. (a) Three-dimensional computed tomography reconstruction of the cervical vertebrae. The white arrow indicates C7. (b) Conventional computed tomography scanning of C7. (A) The posterior tubercle of the transverse process of C7. (c) Ultrasound imaging of C7. The dotted line represents the posterior tubercle of the transverse process of C7. The letters $(\mathrm{A})-(\mathrm{F})$ indicate, respectively, the medial scalenus muscle, posterior tubercle, brachial plexus, sternocleidomastoideus muscle, anterior scalenus muscle, and cervical artery. Post.: posterior position of patients. Ant.: anterior position of patients.

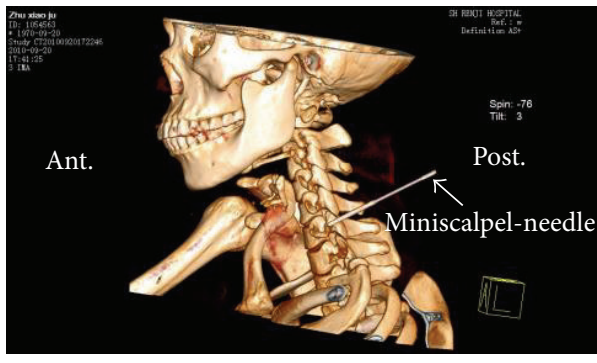

(a)

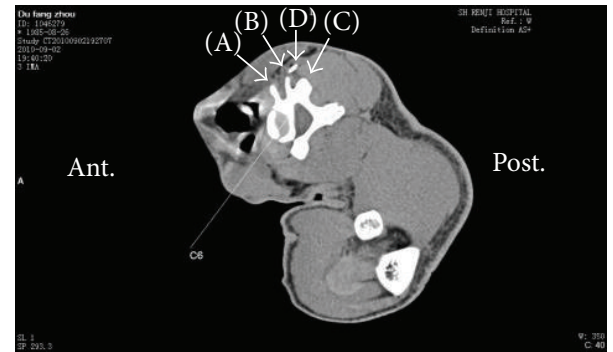

(b)

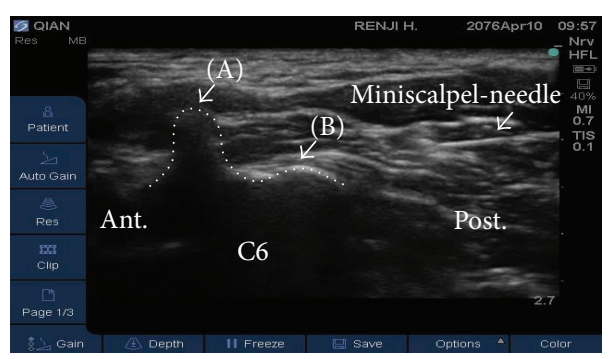

(c)

FIGURE 3: UG-MSN release at the sixth cervical vertebra (C6). (a) Three-dimensional computed tomography reconstruction of the puncture site at C6. The white arrow indicates the MSN. (b) Conventional computed tomography scanning of C6. The letters (A)-(D) indicate, respectively, the anterior tubercle, posterior tubercle, articular process, and the MSN. (c) UG-MSN release at C6, with the MSN clearly indicated. Trigger points showed the following characteristics: hyperechoic skin, adipose tissue of mixed echogenicity, and muscle of hyperechoic, marbled appearance. The dotted line shows the outline of C6. The letters (A) and (B) represent, respectively, the posterior tubercle and articular process. Post.: posterior position of patients. Ant.: anterior position of patients. 
TABLE 1: Baseline characteristics of patients with chronic neck pain before random allocation to be treated with ultrasound-guided MSN release or dry needling.

\begin{tabular}{|c|c|c|c|}
\hline Variable & $\begin{array}{l}\text { UG-MSN release } \\
\quad(n=82)\end{array}$ & $\begin{array}{l}\mathrm{UG}-\mathrm{DN} \\
(n=73)\end{array}$ & $P^{*}$ \\
\hline \multicolumn{4}{|l|}{ Demographics } \\
\hline Age, yr & $39.0 \pm 14.1$ & $42.4 \pm 13.7$ & 0.1 \\
\hline Male, \% & 54.9 & 65.8 & 0.3 \\
\hline Disease duration, yr & $4.7 \pm 2.3$ & $5.3 \pm 2.9$ & 0.1 \\
\hline \multicolumn{4}{|l|}{ Previous treatments, $\%$} \\
\hline Current analgesic medication & 42.7 & 54.8 & 0.2 \\
\hline $\begin{array}{l}\text { Physical therapy, chiropractic, or } \\
\text { massage }\end{array}$ & 47.6 & 58.9 & 0.2 \\
\hline \multicolumn{4}{|l|}{ Pain intensity } \\
\hline 10-point visual analog scale score & $6.8 \pm 1.6$ & $7.1 \pm 1.8$ & 0.3 \\
\hline \multicolumn{4}{|l|}{ Neck disability } \\
\hline Neck disability index score & $17.9 \pm 8.3$ & $16.0 \pm 5.4$ & 0.1 \\
\hline \multicolumn{4}{|l|}{ Health-related quality of life (SF-36) } \\
\hline Physical component score & $41.3 \pm 14.0$ & $43.6 \pm 16.1$ & 0.1 \\
\hline Mental component score & $43.4 \pm 12.6$ & $44.4 \pm 16.8$ & 0.7 \\
\hline
\end{tabular}

UG-DN: ultrasound-guided dry needling; UG-MSN: ultrasound-guided miniscalpel-needle.

Data expressed as mean $\pm \mathrm{SD}$ or $\%$.

${ }^{*}$ Assessed using independent Student's $t$-test for continuous variables and using the $\chi^{2}$ test or Fisher's exact test for categorical variables.

TABLE 2: Longitudinal comparison of primary outcomes in patients with chronic neck pain treated with either UG-MSN release or UG-DN.

\begin{tabular}{|c|c|c|c|}
\hline Outcome & Baseline & 3 months & 6 months \\
\hline \multicolumn{4}{|c|}{ Visual analog scale (VAS) } \\
\hline UG-MSN release & $6.8 \pm 1.6$ & $3.3 \pm 1.2$ & $3.8 \pm 1.2$ \\
\hline UG-DN & $7.1 \pm 1.8$ & $4.2 \pm 1.5$ & $5.8 \pm 1.4$ \\
\hline${ }^{*} P$ value & & $<0.0001$ & $<0.0001$ \\
\hline \multicolumn{4}{|c|}{ Neck disability index (NDI) } \\
\hline UG-MSN release & $17.9 \pm 8.3$ & $6.4 \pm 3.7$ & $7.1 \pm 2.6$ \\
\hline UG-DN & $16.0 \pm 5.4$ & $8.8 \pm 4.5$ & $12.2 \pm 5.5$ \\
\hline${ }^{*} P$ value & & $<0.0001$ & $<0.0001$ \\
\hline \multicolumn{4}{|c|}{ SF-36 physical component score (PCS) } \\
\hline UG-MSN release & $41.3 \pm 14.0$ & $49.2 \pm 14.1$ & $55.1 \pm 19.0$ \\
\hline UG-DN & $43.6 \pm 16.1$ & $44.1 \pm 13.6$ & $47.9 \pm 21.5$ \\
\hline${ }^{*} P$ value & & 0.013 & 0.024 \\
\hline \multicolumn{4}{|c|}{ SF-36 mental component score (MCS) } \\
\hline UG-MSN release & $43.4 \pm 12.6$ & $49.4 \pm 14.6$ & $51.4 \pm 14.5$ \\
\hline UG-DN & $44.4 \pm 16.8$ & $49.0 \pm 15.2$ & $50.8 \pm 16.7$ \\
\hline${ }^{*} P$ value & & 0.778 & 0.801 \\
\hline
\end{tabular}

Data are expressed as mean \pm SD.

* Based on analyses of covariance in which the primary outcome was the response variable and treatment was the explanatory variable; results were adjusted for baseline differences in the response variable.

and improves local microcirculation [25]. In addition to avoiding damage to important adjacent structures, ultrasound imaging could enhance the accuracy of the needle placement and probably contributed to the apparently superior efficacy of the treatment.

DN and MSN have been shown to relieve chronic neck pain $[3,26]$, but proper positioning of trigger points is essential to the success of both techniques and for avoiding severe injuries, including pneumothorax and intercostal nerve damage $[12,13,27]$. Trigger points are sometimes difficult to palpate during physical examination, especially in obese patients, and using ultrasound to guide trigger point injections has been linked to greater therapeutic efficacy and lower possibility of complications [28]. Our results extend these findings by showing for the first time that ultrasound guidance can be used safely and effectively to perform MSN. 


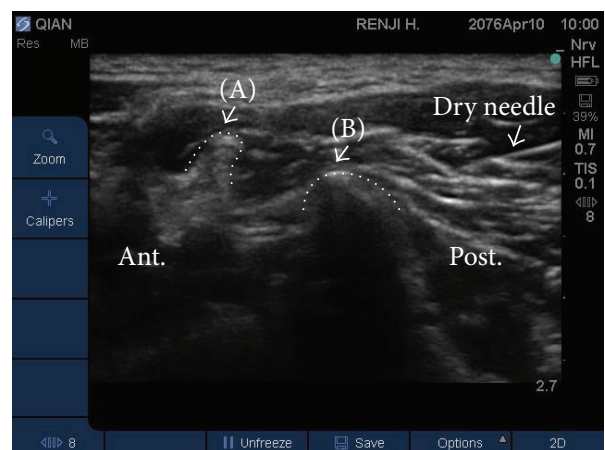

FIGURE 4: UG-DN at the sixth cervical vertebra (C6). The dry needle is clearly indicated. Trigger points showed the same characteristics described in Figure 3. The dotted line shows the outline of C6. The letters $(\mathrm{A})$ and $(\mathrm{B})$ indicate, respectively, the posterior tubercle and articular process. Post.: posterior position of patients. Ant.: anterior position of patients.

Our finding that UG-MSN release is superior to UG$\mathrm{DN}$ is reminiscent of a previous study showing MSN release to be superior to acupuncture in managing myofascial pain syndrome [9]. Our results compared an experimental treatment (UG-MSN) with an active control (UG-DN), and we expect that the therapeutic advantage of UG-MSN would be even greater if a placebo control group had been used. An inactive control group, however, may be unethical under these circumstances, and the debate about ideal controls for acupuncture studies continues [29]. We ruled out using a control group that would receive MSN release without ultrasound guidance because of the risk of severe complications; we also ruled out a control group that would receive UGMSN release outside the trigger points because the resulting lack of efficacy might drastically reduce compliance. Risk of complications led us to rule out control groups treated by other approaches, including acupuncture, steroid injection, and trigger point injection [9-11].

In addition to its randomized and controlled nature, our study fulfills the essential requirement of using wellvalidated outcome measures. While outcomes based on selfreport inherently carry some risk of bias, we believe that outcomes like pain, functional ability, and quality of life can be more accurately assessed by listening to patients rather than physicians.

All treatments were conducted by a single physician in the current study. Such a design helped to ensure consistency between the two treatment groups but decreased the generalizability of the results. Another concern is the completeness of the blinding: patient experience during the procedure may help them to identify which treatment they received. We included only "naïve" subjects in the current study: those who received acupuncture or miniscalpel-needle release previously were not included. Nonetheless, we did not take solid measures to verify the blinding success. As such, the results of the current study need to be interpreted with caution.

\section{Conflict of Interests}

The authors declare that there is no conflict of interests regarding the publication of this paper.

\section{Authors' Contribution}

Yongjun Zheng, Dongping Shi, Xiaotong Wu, Minghong Gu, Zisheng Ai, and Kun Tang contributed equally to this work.

\section{Acknowledgment}

This study was supported by the National Key Basic Research Program (973 Program) of China (2007CB512504).

\section{References}

[1] S. Hogg-Johnson, G. van der Velde, L. J. Carroll et al., "The burden and determinants of neck pain in the general population: Results of the Bone and Joint Decade 2000-2010 Task Force on Neck Pain and Its Associated Disorders," Spine, vol. 33, no. 4, pp. S39-S51, 2008.

[2] J. A. J. Borghouts, B. W. Koes, H. Vondeling, and L. M. Bouter, "Cost-of-illness of neck pain in The Netherlands in 1996," Pain, vol. 80, no. 3, pp. 629-636, 1999.

[3] D. J. Alvarez and P. G. Rockwell, "Trigger points: diagnosis and management," American Family Physician, vol. 65, no. 4, pp. 653-660, 2002.

[4] A. D. Furlan, M. W. van Tulder, D. C. Cherkin et al., "Acupuncture and dry-needling for low back pain," Cochrane Database of Systematic Reviews, vol. 25, no. 1, Article ID CD001351, 2005.

[5] D. Irnich, N. Behrens, J. M. Gleditsch et al., "Immediate effects of dry needling and acupuncture at distant points in chronic neck pain: results of a randomized, double-blind, sham-controlled crossover trial," Pain, vol. 99, no. 1-2, pp. 83-89, 2002.

[6] D. Irnich, N. Behrens, H. Molzen et al., "Randomised trial of acupuncture compared with conventional massage and "sham" laser acupuncture for treatment of chronic neck pain," British Medical Journal, vol. 322, no. 7302, pp. 1574-1578, 2001.

[7] Y.-L. Hsieh, M.-J. Kao, T.-S. Kuan, S.-M. Chen, J.-T. Chen, and C.-Z. Hong, "Dry needling to a key myofascial trigger point may reduce the irritability of satellite MTrPs," American Journal of Physical Medicine and Rehabilitation, vol. 86, no. 5, pp. 397-403, 2007.

[8] E. A. Tough, A. R. White, T. M. Cummings, S. H. Richards, and J. L. Campbell, "Acupuncture and dry needling in the management of myofascial trigger point pain: a systematic review and meta-analysis of randomised controlled trials," European Journal of Pain, vol. 13, no. 1, pp. 3-10, 2009.

[9] C. Ma, S. Wu, G. Li, X. Xiao, M. Mai, and T. Yan, "Comparison of miniscalpel-needle release, acupuncture needling, and stretching exercise to trigger point in myofascial pain syndrome," The Clinical Journal of Pain, vol. 26, no. 3, pp. 251-257, 2010.

[10] C. Wang, Z. Xiong, C. Deng, W. Yu, and W. Ma, "Miniscalpelneedle versus trigger-point injection for cervical myofascial pain syndrome: a randomized comparative trial," Journal of Alternative and Complementary Medicine, vol. 13, no. 1, pp. 1416, 2007.

[11] M. Chao, S. Wu, and T. Yan, "The effect of miniscalpel-needle versus steroid injection for trigger thumb release," Journal of 
Hand Surgery: European Volume, vol. 34, no. 4, pp. 522-525, 2009.

[12] Y. Ding, Y. Duan, Y. Li, and L. Zhang, “The analysis of complications caused by the treatment of tenosynovitis by miniscalpelneedle," Journal of Hebei North University (Medical Edition), vol. 24, pp. 67-68, 2007.

[13] Y. Zhang and C. Guo, "Discussion on security of acupotomology," Chinese Archives of Traditional Chinese Medicine, vol. 29, pp. 2214-2215, 2011.

[14] K. Galiano, A. A. Obwegeser, G. Bodner, R. Schatzer, M. Schocke, and H. Gruber, "Ultrasound is not the only technique to visualize third occipital nerve blockade," Anesthesiology, vol. 105, no. 4, p. 858, 2006.

[15] K. Galiano, A. A. Obwegeser, C. Walch, R. Schatzer, F. Ploner, and H. Gruber, "Ultrasound-guided versus computed tomography-controlled facet joint injections in the lumbar spine: a prospective randomized clinical trial," Regional Anesthesia and Pain Medicine, vol. 32, no. 4, pp. 317-322, 2007.

[16] S. N. Narouze, A. Vydyanathan, L. Kapural, D. I. Sessler, and N. Mekhail, "Ultrasound-guided cervical selective nerve root block: a fluoroscopy-controlled feasibility study," Regional Anesthesia and Pain Medicine, vol. 34, no. 4, pp. 343-348, 2009.

[17] L. Lepage, D. G. Altman, K. F. Schulz et al., "The revised CONSORT statement for reporting randomized trials: explanation and elaboration," Annals of Internal Medicine, vol. 134, no. 8, pp. 663-694, 2001.

[18] E. C. Huskisson, "Measurement of pain," The Lancet, vol. 2, no. 7889, pp. 1127-1131, 1974.

[19] S. Narouze, "Sonoanatomy of the cervical spinal nerve roots: implications for brachial plexus block," Regional Anesthesia and Pain Medicine, vol. 34, no. 6, p. 616, 2009.

[20] H. Vernon and S. Mior, "The neck disability index: a study of reliability and validity," Journal of Manipulative and Physiological Therapeutics, vol. 14, pp. 409-415, 1991.

[21] S. Wu, C. Ma, M. Mai, and G. Li, "Translation and validation study of Chinese versions of the neck disability index and the neck pain and disability scale," Spine, vol. 35, no. 16, pp. 15751579, 2010.

[22] J. E. Ware Jr. and C. D. Sherbourne, “The MOS 36-item shortform health survey (SF-36). I. Conceptual framework and item selection," Medical Care, vol. 30, no. 6, pp. 473-483, 1992.

[23] L. Li, H. M. Wang, and Y. Shen, "Chinese SF-36 Health Survey: translation, cultural adaptation, validation, and normalisation," Journal of Epidemiology and Community Health, vol. 57, no. 4, pp. 259-263, 2003.

[24] B. Ma and K. Li, Medical Statistics, People's Medical Publishing House, Beijing, China, 2008.

[25] J. N. Ghia, W. Mao, T. C. Toomey, and J. M. Gregg, "Acupuncture and chronic pain mechanisms," Pain, vol. 2, no. 3, pp. 285-299, 1976.

[26] N. A. Scott, B. Guo, P. M. Barton, and R. D. Gerwin, "Trigger point injections for chronic non-malignant musculoskeletal pain: a systematic review," Pain Medicine, vol. 10, no. 1, pp. 5469, 2009.

[27] D. Kalawokalani, "Malpractice claims for non-operative pain management: a growing pain for anesthesiologists?" ASA Professional Information, vol. 63, no. 6, pp. 16-18, 1999.

[28] K. P. Botwin, K. Sharma, R. Saliba, and B. C. Patel, "Ultrasoundguided trigger point injections in the cervicothoracic musculature: a new and unreported technique," Pain Physician, vol. 11, no. 6, pp. 885-889, 2008.
[29] F. Dincer and K. Linde, "Sham interventions in randomized clinical trials of acupuncture-a review," Complementary Therapies in Medicine, vol. 11, no. 4, pp. 235-242, 2003. 


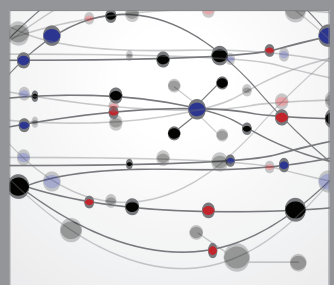

The Scientific World Journal
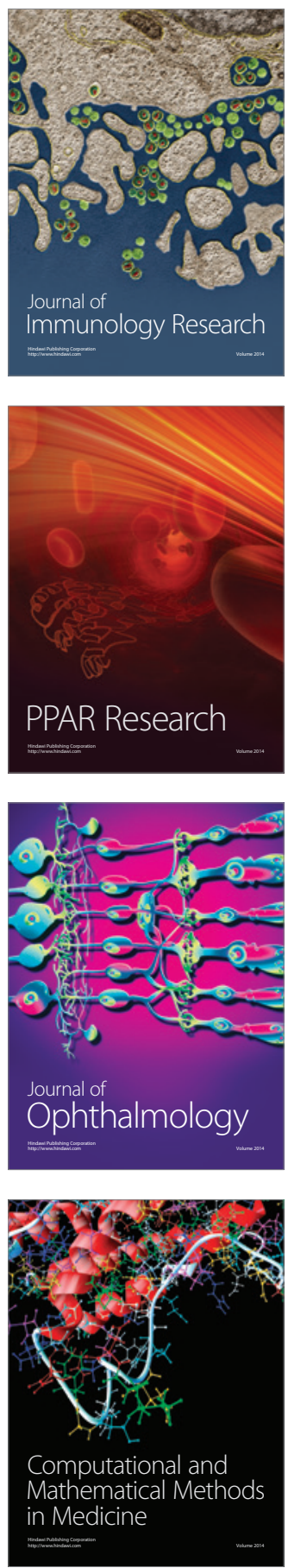

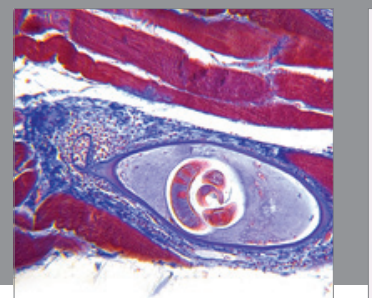

Gastroenterology

Research and Practice
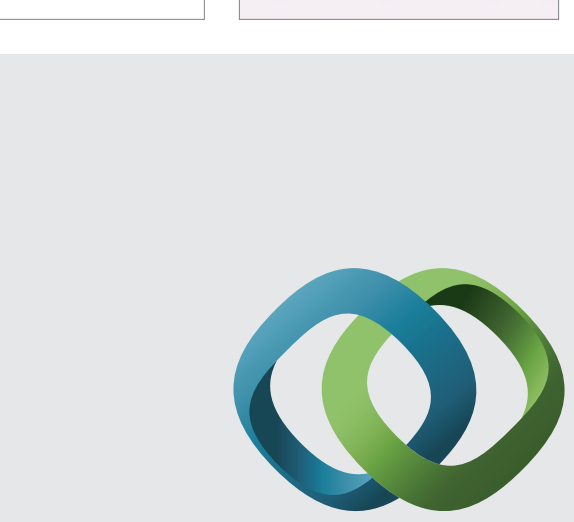

\section{Hindawi}

Submit your manuscripts at

http://www.hindawi.com
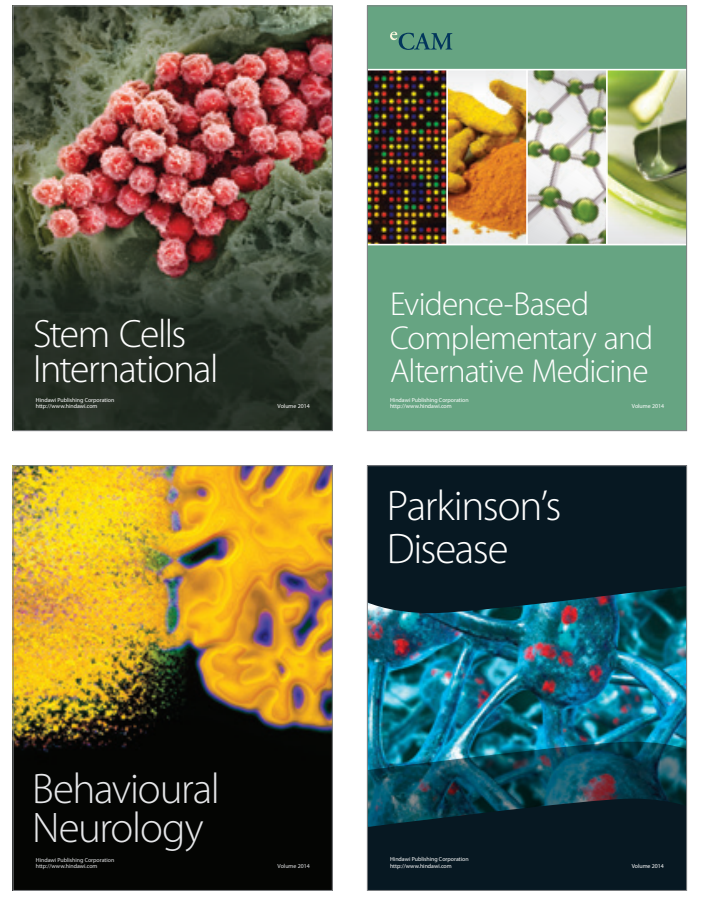
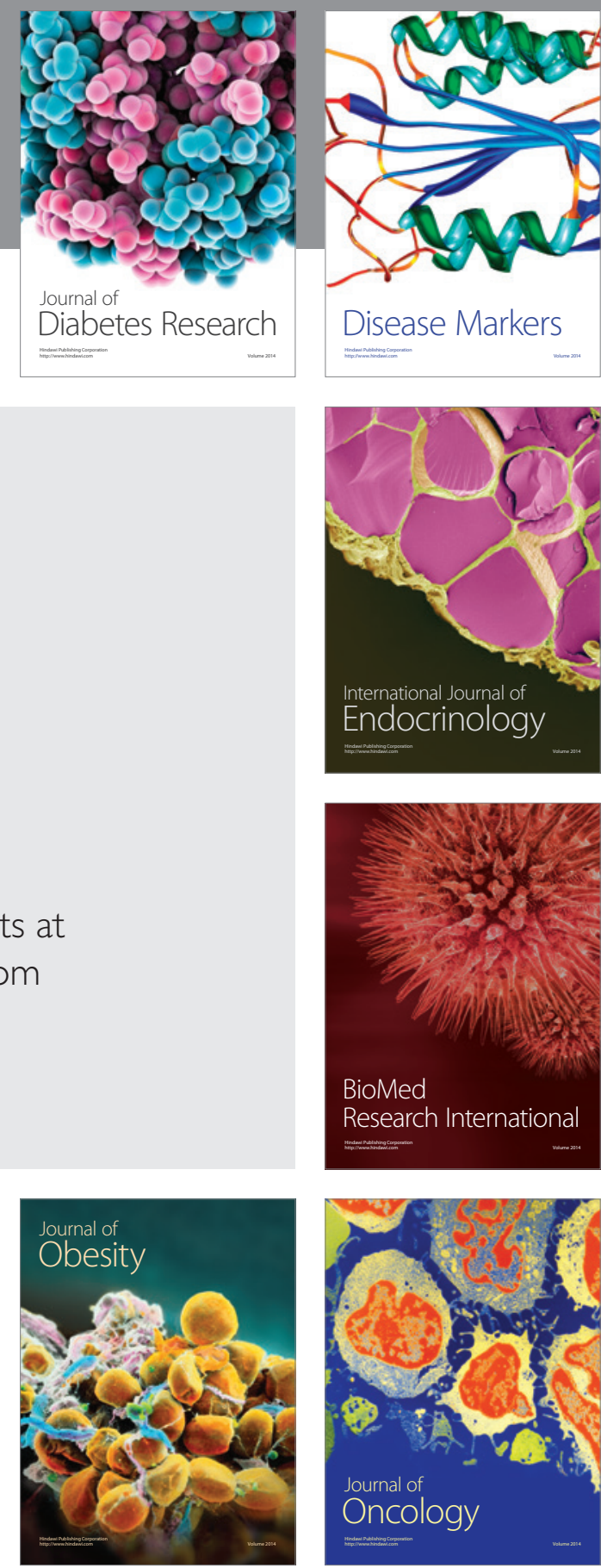

Disease Markers
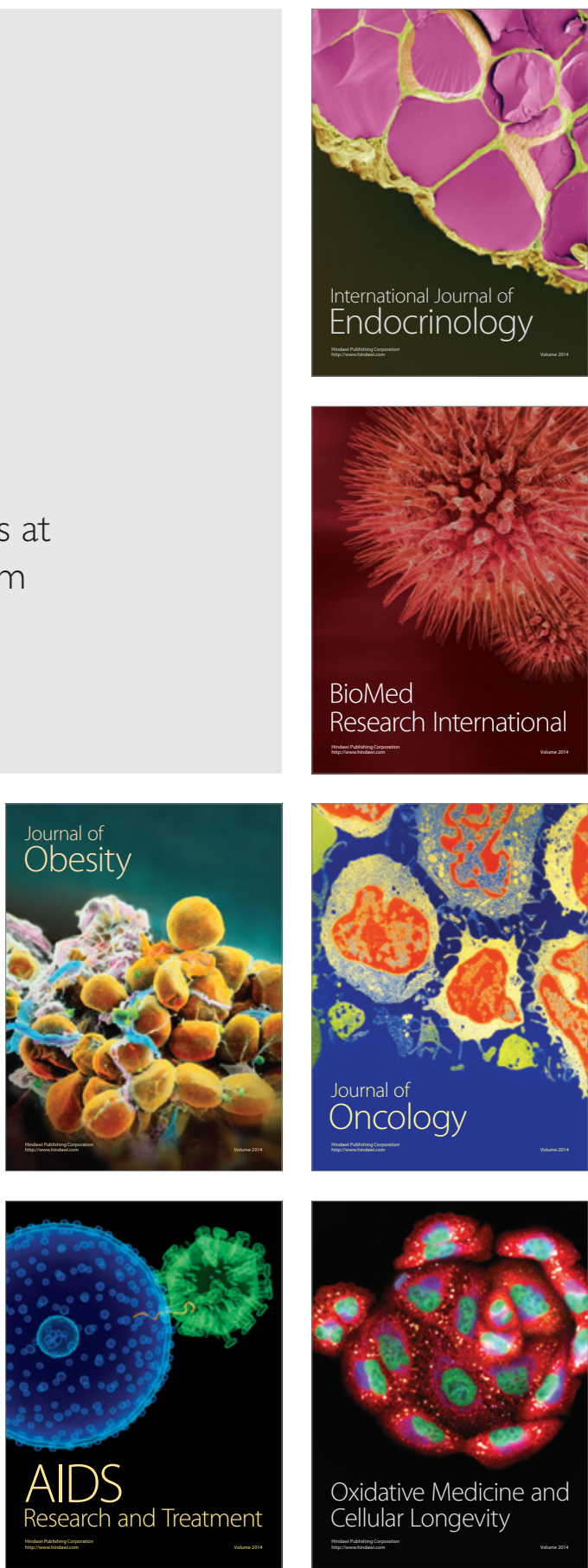